\title{
Influence of Cold Ischemia Time on RNA Quality of HCC Frozen Tissues and Matched Adjacent Tissues.
}

Yue Hu, Yanhong Liu , Hong Gao, Meiling Ge, Jie Ding, Qing Ye*

Biobank of Nanjing Drum Tower Hospital, The Affiliated Hospital of Nanjing University Medical School, Nanjing, Jiangsu, China. Nanjing Multi-center Biobank, Nanjing Public Health Bureau, Nanjing , Jiangsu, China. Jiangsu Biobank of Clinical Resources, Nanjing , Jiangsu, China.

\section{Introduction}

RNA degradation is a major problem in tissue banking. Standard Pre-analytical code (SPREC) can manage and trace the influence factors of pre-analytical effectively, improve the quality of samples, and improve the accuracy of experimental results. The SPREC variables include that sample types, collecting methods, warm ischemia time, cold ischemia time, fixed type, storage time and long-time storage conditions, etc. For the frozen tissue, cold ischemia time probably be the most important factors which influence the quality of RNA. This paper mainly research on the influence of cold ischemia time on RNA quality of hepatocellular carcinoma frozen tissues.

\section{Materials \& Methods}

The sample collection procedure of HCC frozen tissues:

\section{Sample received:}

$>$ Check and receive the surgical resection sample, blood sample, informed consent and mode pattern.

\section{Sample dissection:}

$>$ Estimate the location of tumor in the liver according to the mode pattern.

$>$ Clean up the sample, confirm the location of cutting edge and capsule.
> Apply the ink on the cutting edge and pay attention to dry ink with absorbent paper.

$>$ Perpendicular to the liver cut edge surface and cut the liver parallel, with the interval of $1 \mathrm{~cm}$.

\section{Taking pictures:}

$>$ Prepare the black background and ruler, and arrange the liver section in a single row or double row in the same direction.

$>$ Take overall, local and flip side photos. Sample collection:

$>$ Collecting the tumor tissues and matched adjacent tissues.

RNA quality control:

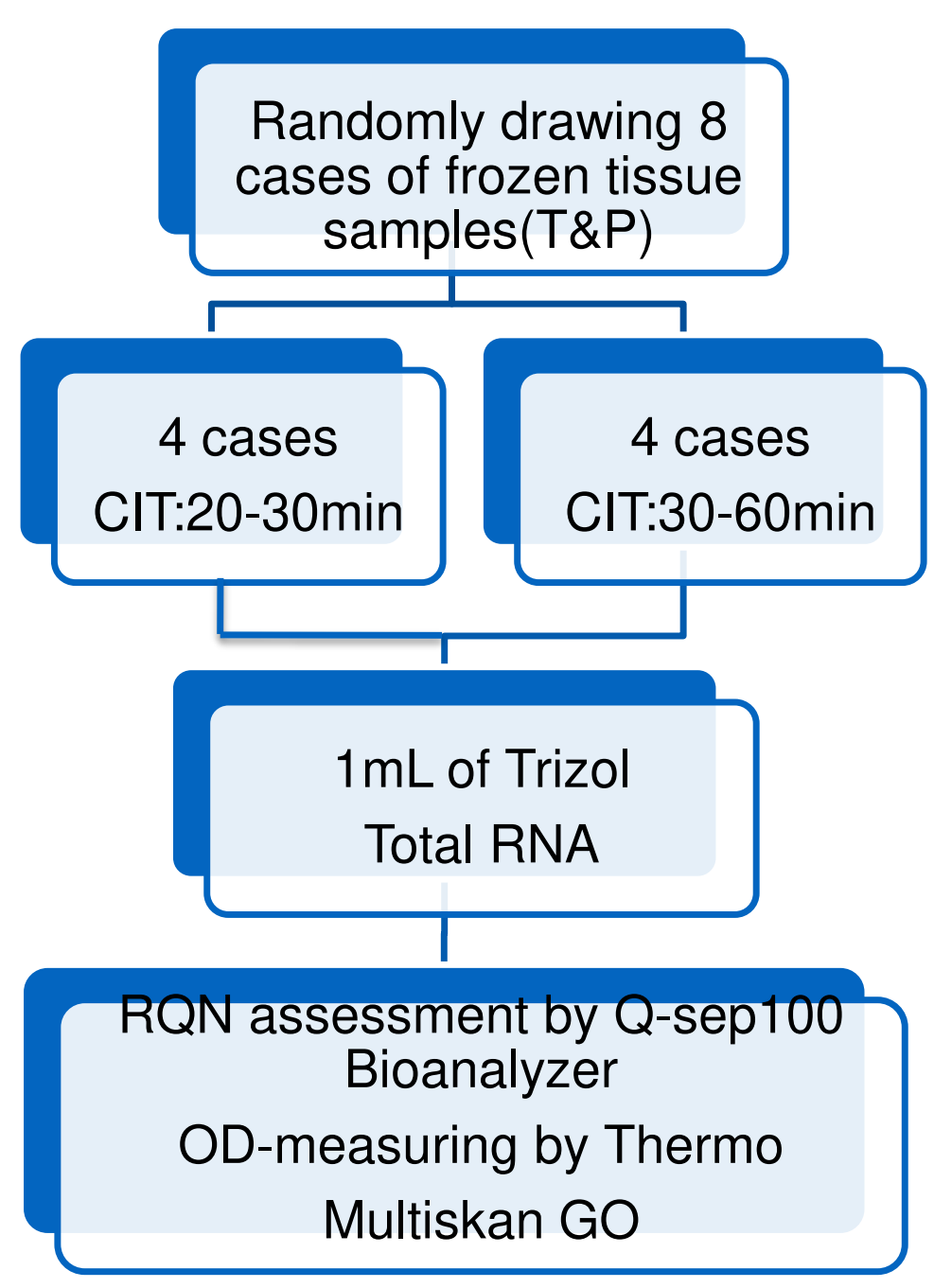

\section{Results}

$\checkmark$ Sample dissection and take photos:

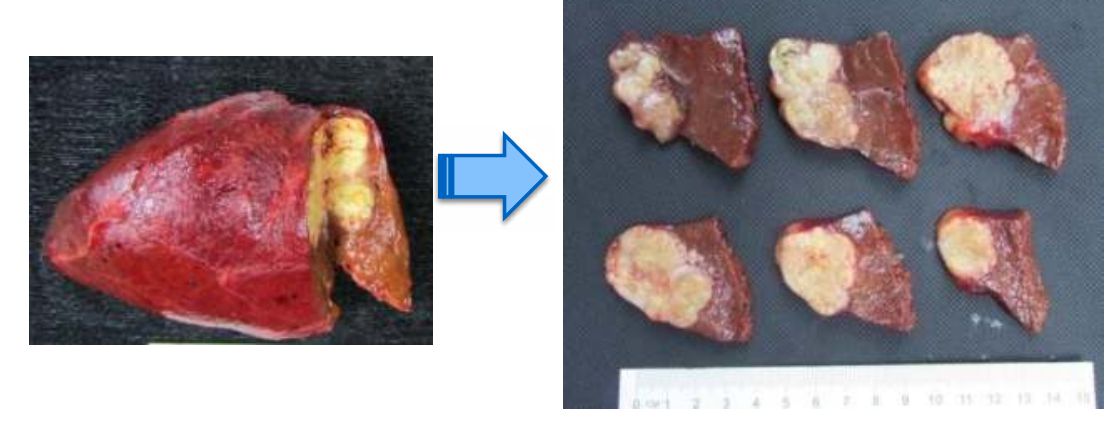

Figure 1 HCC dissection

- RNA quality control:

All the OD260/OD280 and OD260/OD230 ratios are between 1.9 and 2.1, conform to the requirements of RNA purity. When the cold ischemia time is 20 to 30 minutes, the integrity of RNA extracted from all the tissues was qualified (RQN scores $>8$ ). While the cold ischemia time is 30 to 60 minutes, the RQN scores $>6$. We also calculated the $28 \mathrm{~s}: 18 \mathrm{~s}$. Using a cutoff ratio of 0.6 , all of them were qualified (Table 1 and Figure 2).
४ Pathological morphological quality control: In addition, all tissue sections $(3 \mu \mathrm{m})$ were cut and stained with hematoxylin and eosin to assess morphology. According to the HE staining results, the percentage of viable tumor area $>80 \%$ in tumor tissues, and tumor cell infiltration were not found in matched adjacent tissues.

\section{Conclusion}

In our study, the integrity of RNA extracted from HCC tissues was significantly influenced by cold ischemia time. The RQN scores are better when the cold ischemia time is less than 30 minutes. But the cold ischemia time did not adversely influence morphology. The integrity of RNA has no significant difference between tumor tissues and matched adjacent tissues. In the process of sample collection in biobank, in order to guarantee the quality of RNA, the cold ischemia time should be within 30 minutes.

Key words: Hepatocellular carcinoma,
Table1 RNA concentration, purity and integrity
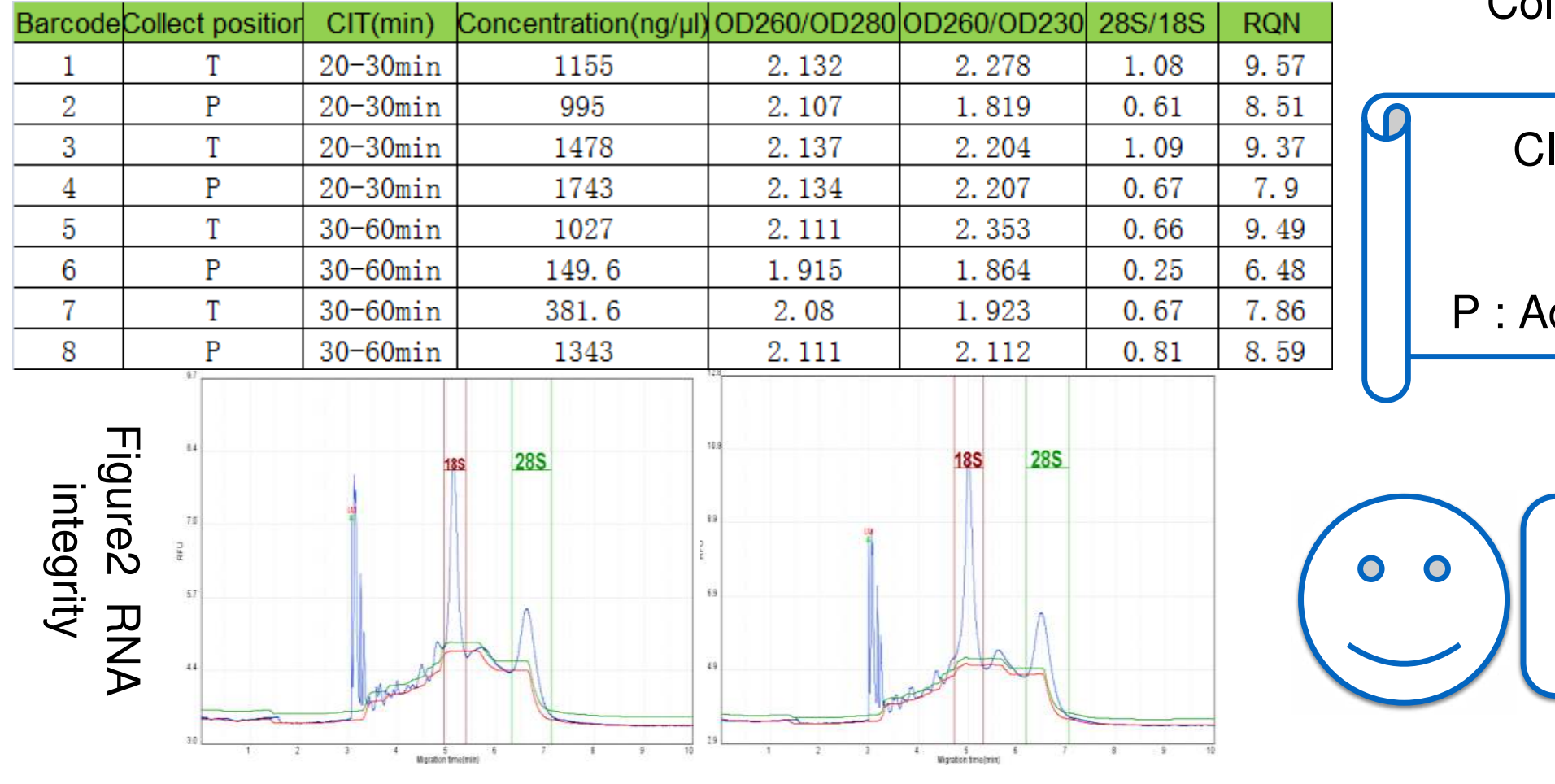
Cold ischemia time, RNA quality.

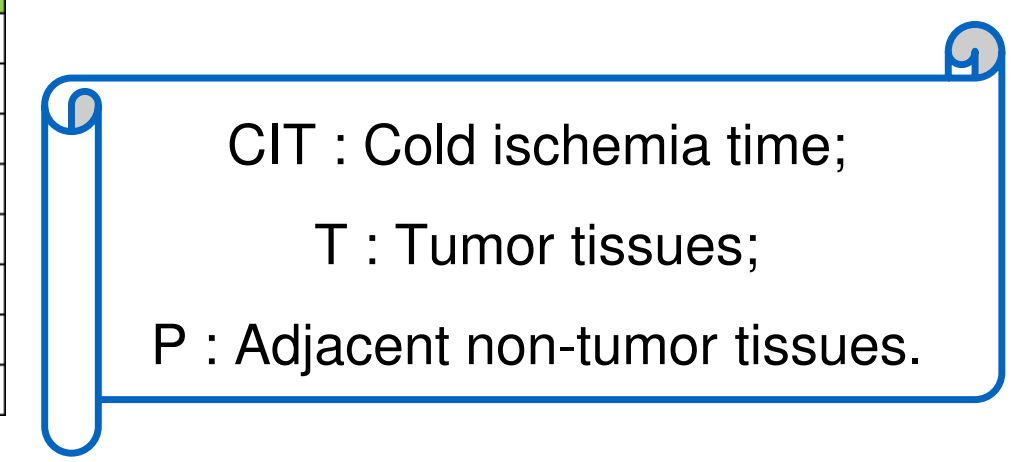

\begin{tabular}{c} 
*Corresponding author \\
Email: \\
nanjingbiobank@163.com \\
\hline
\end{tabular} 